\title{
FIRST DNA BARCODING AND NEW RECORDS OF THE MEDITERRANEAN CADDISFLY SPECIES MICROPTERNA WAGENERI MAL. (TRICHOPTERA, LIMNEPHILIDAE) IN CROATIA WITH NOTE ON DNA BARCODING AND DIVERSITY OF GENUS MICROPTERNA IN CROATIA
}

\section{Mladen Kučinić ${ }^{1}$, AnĐela Ćukušić ${ }^{2}$, Sanja Žalac ${ }^{3}$, Martina Podnar ${ }^{4}$, Kanat Kambarovich Akhmetov ${ }^{5}$, Nazymgul Akimbekova ${ }^{5}$, Sholpan Moldazhanovna Zhumadina ${ }^{5}$ \& Ivan VučKović ${ }^{6}$}

\author{
${ }^{1}$ Department of Biology (Laboratory for Entomology), Faculty of Science, University of Zagreb, \\ Rooseveltov trg 6, 10000 Zagreb, Croatia \\ ${ }^{2}$ Geonatura Ltd. Consultancy in Nature Protection, Fallerovo šetalište 22, 10000 Zagreb, Croatia \\ ${ }^{3}$ Plitvička jezera National park, 53231 Plitvička jezera, Croatia \\ ${ }^{4}$ Croatian Natural History Museum, Demetrova 1, 10000 Zagreb, Croatia \\ ${ }^{5}$ S. Toraigyrov State University in Pavlodar, Lomov St 64, Pavlodar 140000, Kazakhstan \\ ${ }^{6}$ Elektroprojekt d.d., Civil and Architectural Engineering Department, Water Resources, \\ Nature and Environmental protection, Alexandera von Humboldta 4, 10000 Zagreb, Croatia
}

Kučinić, M., Ćukušić, A., Žalac, S., Podnar, M., Kambarovich Akhmetov, K., Akimbekova, N., Moldazhanovna Zhumadina, S. \& Vučković, I.: First DNA barcoding and new records of the Mediterranean caddisfly species Micropterna wageneri Mal. (Trichoptera, Limnephilidae) in Croatia with note on DNA barcoding and diversity of genus Micropterna in Croatia. Nat. Croat., Vol. 26, No. 1, 81-98, 2017, Zagreb.

The paper brings data about the new findings of the rare Mediterranean caddisfly species Micropterna wageneri Mal. in Croatia with first data of DNA barcoding for this species. The species is recorded in Konavle region and on the Mt. Biokovo. We also present the diversity of the genus Micropterna in Croatia and discuss issues related to the DNA barcoding for this genus in Croatia and Europe.

Key words: Trichoptera, Micropterna, DNA barcoding, museum collections, Croatia, Biokovo, Konavle

Kučinić, M., Ćukušić, A., Žalac, S., Podnar, M., Kambarovich Akhmetov, K., Akimbekova, N., Moldazhanovna Zhumadina, S. \& Vučković, I.: Prvo DNA barkodiranje i novi nalazi sredozemne vrste tulara Micropterna wageneri Mal. (Trichoptera, Limnephilidae) u Hrvatskoj, s osvrtom na DNA barkodiranje i raznolikost roda Micropterna u Hrvatskoj. Nat. Croat., Vol. 26, No. 1, 81-98, 2017, Zagreb.

Rad donosi podatke o novim nalazima rijetke mediteranske vrste tulara Micropterna wageneri Mal. u Hrvatskoj, s prvim podacima o DNA barkodiranju te vrste. Vrsta je zabilježena u Konavlima i na Biokovu. Također se predstavlja raznolikost roda Micropterna u Hrvatskoj i raspravlja o DNA barkodiranju tog roda u Hrvatskoj i Europi.

Ključne riječi: Trichoptera, Micropterna, DNA barkodiranje, muzejske zbirke, Hrvatska, Biokovo, Konavle 


\section{INTRODUCTION}

Identification of various animal species is a starting point of research into the morphology, histology, genetics, physiology, distribution, ecology, phylogenetics and other biological characteristics of individual species. Such approach in biology is based on the rules of binomial nomenclature set up by Carl Linnaeus in the $18^{\text {th }}$ century (LinneAus, 1758). Morphological characteristics have for a long time been used as the basis for determination of organisms and this practice is being used until today. Determination of populations to the level of species enables us to understand the characteristics of fauna, its diversity and biogeography within a certain area. Due to a high complexity of diversity of numerous groups of animals, in particular invertebrates, many of which are similar, sibling or cryptic species, other methods are used to support a more exact determination of species and finding those that cannot be determined by conventional morphological methods (Ross, 1974; BickFord et al., 2007: Dincă et al., 2013, 2016; Previšić et al.,2014). One of the recent and most frequently used methods in analysis of biodiversity and determination of organisms within a particular area is the DNA barcoding method (HeвERT et al., 2003a, 2003b). This method is used to identify animal species and is based on sequencing of the standardized segment of the mitochondrial (mt) cytochrome c oxidase subunit 1 (COI) gene (НевERT et al., 2003a, 2003b).

Research of caddisflies in Croatia became more intensive in the second half of the $20^{\text {th }}$ century through a larger number of limnological studies (MatoničKIN et al., 1971; MatoničKin, 1987; MatoničKIn \& Pavletić, 1967; Habdija, 1989) and continued with increased intensity during the last two decades with numerous taxonomic, faunal and ecological studies (e.g. Krulik, 1979; Kučinić \& Ilić, 1993; Kučinić, 2002; Kučinić \& Malicky, 2002; Habdija et al., 2004; Previšrć et al., 2007, 2009, 2014; Kučinić et al., 2008, 2011; VučKović et al., 2011; Szivák et al., 2013; Matić et al., 2016). Application of the DNA barcoding method in these studies has started recently in taxonomic and faunistic studies of Croatian Trichoptera (Kučinić et al., 2013, 2016).

This paper presents the new finding of the rare Mediterranean caddisfly species Micropterna wageneri Malicky 1971 in Croatia with first data of DNA barcoding for this species. We also present the diversity of the genus Micropterna Stein in Croatia and discuss issues related to the DNA barcoding for this genus in Croatia and Europe.

\section{MATERIAL AND METHODS}

\section{Fieldwork and research areas}

Our research took place in the Konavle region and in the Nature Park Biokovo. During our investigation in the Konavle region (valley) we collected caddisfly specimens on several locations on springs, streams and the River Ljuta. Konavle region is located in the southern part of Croatia (Dalmatia region). In this part of Croatia there are only few permanent streams and rivers and some temporary streams. Very interesting aquatic habitat in Konavle valley are small manmade canals. We visited Konavle valley three times during 2015 and one time during 2016.

Nature Park Biokovo is situated in central Dalmatia. It is very impressive mountain area with lot of endemic species of animals and plants (e.g. CASALE \& Jalžıć, 1988). The highest peak is Sv. Jure with $1762 \mathrm{~m}$ above sea level. Except several small springs and streams on south expositions on lower elevation, in the highest area of Biokovo there 
are no natural aquatic habitats. During 2015 we visited Nature Park Biokovo three times and one time during May of 2017.

Adults of caddisflies were collected using UV light traps during night and with entomological net during day. The specimens were stored in $96 \%$ alcohol. After determination all caddisfly specimens collected during 2015 were deposited in the Trichoptera collection in the Croatian Natural History Museum in Zagreb. NIP Trichoptera collection is one of the results of the NATURA 2000 Integration Project (NIP). Specimens collected in 2016 were deposited in the Trichoptera collection Vučković and in the Trichoptera collection Kučinić.

For determination of species from the genus Micropterna we used standard literature Kumanski (1988) and Malicky (2004). Systematic presentation follows Malicky (2004) and Morse (2017).

\section{DNA extraction, PCR amplification and phylogenetic analysis}

Genomic DNA was extracted from legs of nine specimens, two M. wageneri and other caddisflies listed in Tab. 1 with specimen ID marked with bold letters. All specimens used in this study are kept as voucher specimens in the Trichoptera DNA Barcode Collection in the Croatian Natural History Museum in Zagreb. Genomic DNA was extracted using GenElute Mammalian Genomic DNA Miniprep kit (Sigma-Aldrich, Germany) according to the manufacturer's specifications and eluted in $100 \mu \mathrm{l}$ of elution buffer. For the amplification of the COI-5P barcode region LCO1490 and HCO2198 (Folmer et al., 1994) primers were used. The volume of mixture for polymerase chain reactions (PCR) was $50 \mu \mathrm{l}$. The PCR mixture contained 1 x Go Taq®Reaction Buffer (containing $1.5 \mathrm{mM} \mathrm{MgCl} 2$, Promega), $0.2 \mathrm{mM}$ of each dNTP, $0.4 \mu \mathrm{M}$ of each primer, 1.25 units of Go Taq®DNA Polymerase (Promega) and $5 \mu$ l of DNA eluate. PCR cycling conditions comprised an initial denaturation step $\left(94^{\circ} \mathrm{C}\right.$ for $\left.2 \mathrm{~min}\right)$ followed by 35 cycles of denaturation at $94^{\circ} \mathrm{C}$ for $30 \mathrm{~s}$, annealing at $50^{\circ} \mathrm{C}$ for $30 \mathrm{~s}$ and elongation at $72^{\circ} \mathrm{C}$ for $90 \mathrm{~s}$, and a final extension step of $72^{\circ} \mathrm{C}$ for $7 \mathrm{~min}$. Product purification and bidirectional sequencing was performed by Macrogen Inc. sequencing service (Seoul, South Korea) using the amplification primers. Sequences were edited manually and aligned using the program BioEdit (HALL, 1999).

DNA sequences obtained in this study were submitted to Barcode of Life Data Systems (BOLD, Ratnasingham \& Hebert, 2007; Tab. 1). For phylogenetic analysis all available Micropterna DNA barcode sequences were retrived from the BOLD System database (BOLD IDs are given in Tab. 1). As outgroups we selected four species belonging to different genera of the family Limnephilidae, such as genus Mesophylax McLachlan; Potamophylax Wallengren; Allogamus Schmid; Halesus Stephens (Tab. 1). Identical sequences were collapsed into unique haplotypes using FaBox v.1.41 (VILleSEN, 2007). All haplotypes are listed in Tab. 1. Two different methods of tree reconstruction were used: Neighbor-Joining (NJ) and Maximum likelihood (ML) as implemented in MEGA 7.0. (KumAr et al., 2016) to infer phylogeny-based specimens identification. Details of phylogenetic analyses were the same as in Kučinić et al. (2016).

Inter- and intraspecific genetic uncorrected pairwise divergences ( $p$-distances) were calculated in MEGA 7.0. (KumAR et al., 2016). The number of hypothetical species within data set was estimated based on barcode gap (difference between inter- and intraspecific genetic distances) by using Automatic Barcode Gap Discovery, ABGD (Puillandre et al., 2012). The mtCOI data set was submitted to the ABGD online website using the same settings as described in Kučinić et al. (2016). 


\begin{tabular}{|c|c|c|c|c|c|c|c|c|c|c|c|c|}
\hline & BOLD Sequence ID & 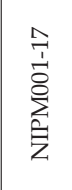 & 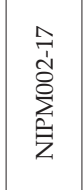 & 空 & 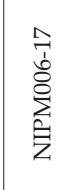 & 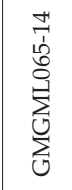 & 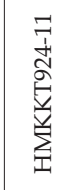 & 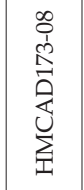 & 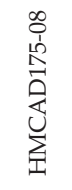 & 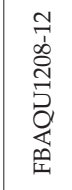 & 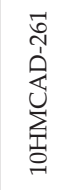 & 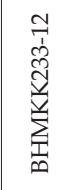 \\
\hline & Specimen ID & $\sum_{i=1}^{J 1}$ & 柋 & $\sum_{i=}^{U^{\prime}}$ & 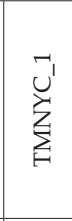 & 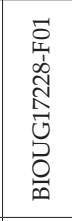 & 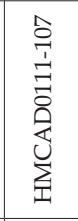 & 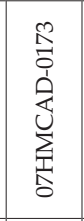 & 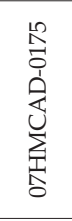 & 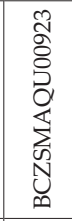 & 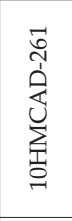 & 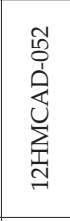 \\
\hline 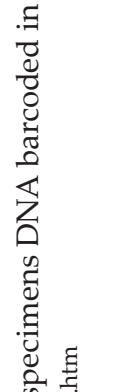 & Location & 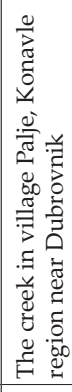 & 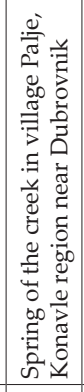 & 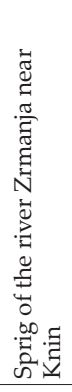 & 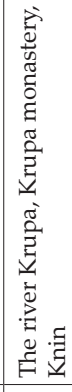 & 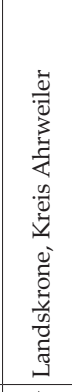 & 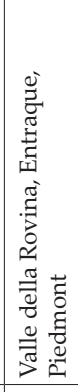 & 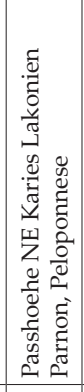 & 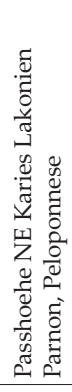 & 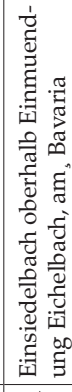 & 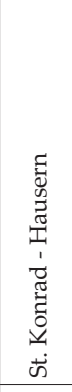 & 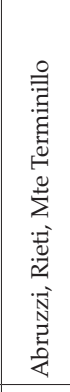 \\
\hline 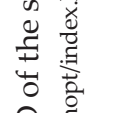 & Country & 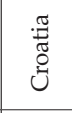 & 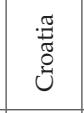 & 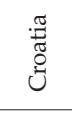 & 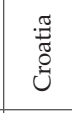 & 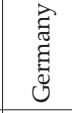 & 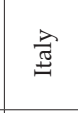 & $\begin{array}{l}\ddot{\Xi} \\
\text { Uू }\end{array}$ & $\begin{array}{l}\mathscr{\Xi} \\
\stackrel{\Xi}{0}\end{array}$ & $\begin{array}{l}\text { है } \\
\text { हूँ } \\
\text { Uू }\end{array}$ & 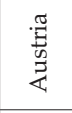 & 无 \\
\hline 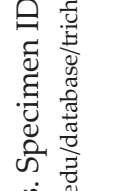 & $\begin{array}{l}\text { Specimen morphologi- } \\
\text { cal identification by: }\end{array}$ & 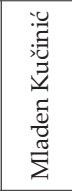 & 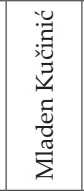 & 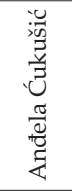 & 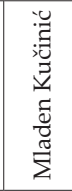 & 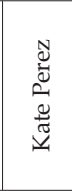 & 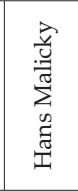 & 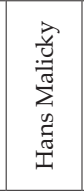 & 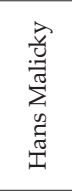 & 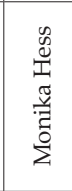 & 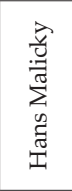 & 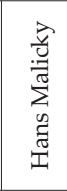 \\
\hline $\begin{array}{l}0 \\
0 \\
0 \\
\text { हू. }\end{array}$ & Sex & 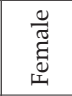 & 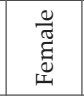 & $\frac{0}{\frac{0}{\pi}}$ & $\sum_{\frac{\pi}{\pi}}^{\frac{0}{\pi}}$ & a. & $\sum_{\pi}^{\frac{0}{\sigma}}$ & $\frac{\pi}{\pi}$ & $\frac{0}{\frac{0}{\pi}}$ & a. & c. & $\sum^{\frac{0}{\pi}}$ \\
\hline 3 & Life Stage & $\begin{array}{l}\frac{\vec{z}}{\mathrm{z}} \\
\frac{0}{4}\end{array}$ & $\begin{array}{l}\frac{\overrightarrow{5}}{0} \\
\frac{0}{4}\end{array}$ & $\frac{\vec{z}}{\frac{3}{4}}$ & $\begin{array}{l}\frac{\vec{T}}{\mathrm{~T}} \\
\frac{0}{4}\end{array}$ & a. & $\frac{\overrightarrow{5}}{\frac{\mathrm{g}}{4}}$ & $\frac{\overrightarrow{3}}{\frac{7}{4}}$ & $\frac{\vec{J}}{\frac{7}{4}}$ & $\stackrel{\widetilde{\Xi}}{\Xi}$ & $\frac{\vec{T}}{\frac{7}{4}}$ & $\begin{array}{l}\frac{\vec{J}}{\vec{z}} \\
\frac{0}{4}\end{array}$ \\
\hline 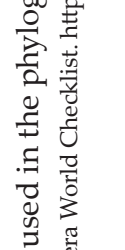 & $\begin{array}{l}\text { Synonim according to } \\
\text { Morse * }\end{array}$ & & & & 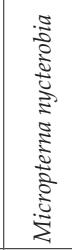 & 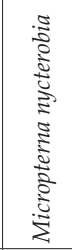 & 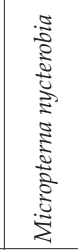 & 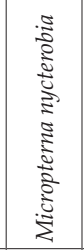 & 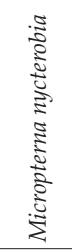 & 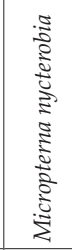 & 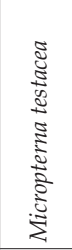 & \\
\hline 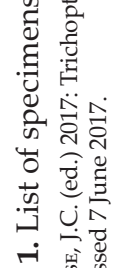 & $\begin{array}{r}\text { Taxonomic designation } \\
\text { according to identifier }\end{array}$ & 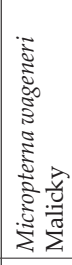 & 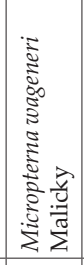 & 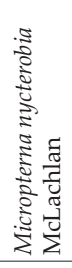 & 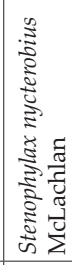 & 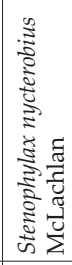 & 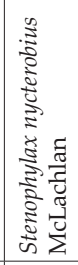 & 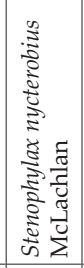 & 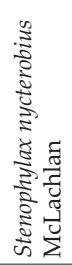 & 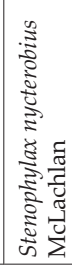 & 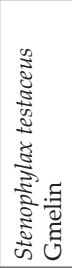 & 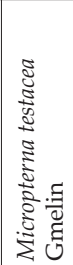 \\
\hline 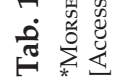 & mt $C O I$ haplotype & $\bar{E}$ & $\hat{~}$ & $\hat{I}^{m}$ & 焉 & $\begin{array}{l}\text { மn } \\
\hat{\mid}\end{array}$ & 焉 & $\hat{\mid}$ & $\begin{array}{l}\infty \\
\hat{1}\end{array}$ & 主 & $\begin{array}{l}\stackrel{\rho}{1} \\
\text { 主 }\end{array}$ & $\begin{array}{l}F \\
\text { I }\end{array}$ \\
\hline
\end{tabular}




\begin{tabular}{|c|c|c|c|c|c|c|c|c|c|c|c|}
\hline BOLD Sequence ID & 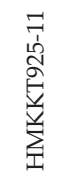 & 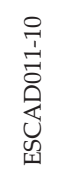 & 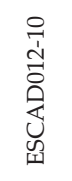 & 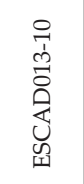 & 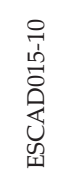 & 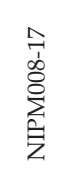 & 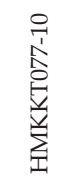 & 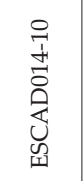 & 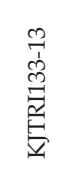 & 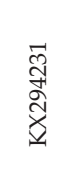 & 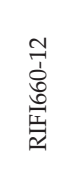 \\
\hline Specimen ID & 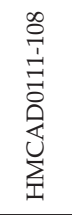 & 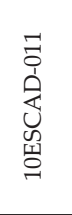 & 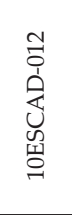 & 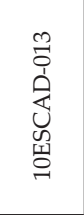 & 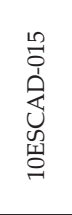 & 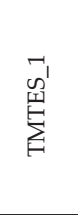 & 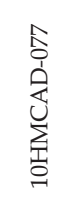 & 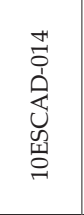 & 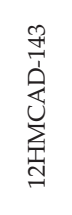 & 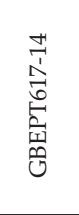 & 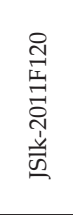 \\
\hline Location & 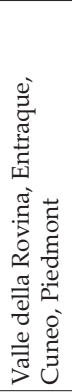 & 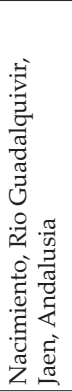 & 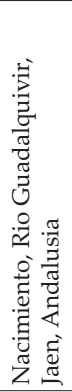 & 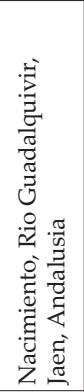 & 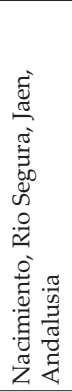 & 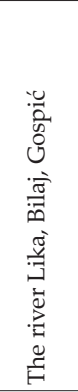 & 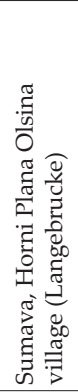 & 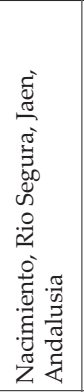 & 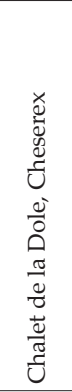 & 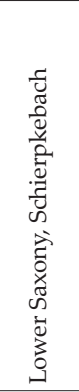 & 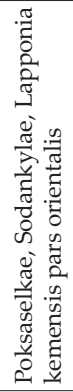 \\
\hline Country & $\stackrel{\vec{\Xi}}{ \pm}$ & $\begin{array}{l}\text { जี } \\
\text { के }\end{array}$ & $\begin{array}{l}\text { है } \\
\text { की }\end{array}$ & $\begin{array}{l}\text { ज్ } \\
\text { के }\end{array}$ & $\begin{array}{l}\text { : } \\
\text { की }\end{array}$ & 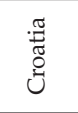 & 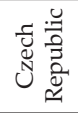 & $\begin{array}{l}\text { हू } \\
\text { के }\end{array}$ & 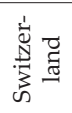 & 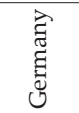 & 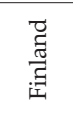 \\
\hline $\begin{array}{l}\text { Specimen morphologi- } \\
\text { cal identification by: }\end{array}$ & 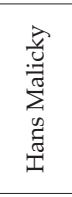 & 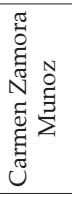 & 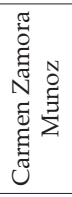 & 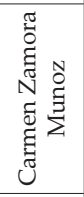 & 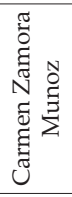 & 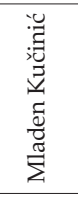 & 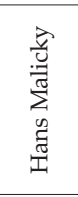 & 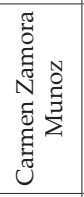 & 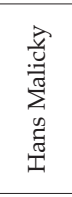 & 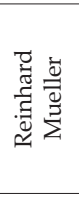 & 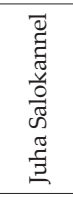 \\
\hline Sex & $\frac{0}{\Sigma^{\pi}}$ & 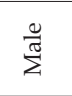 & $\frac{0}{\pi^{\pi}}$ & $\frac{\pi}{\frac{\pi}{\pi}}$ & $\frac{\pi}{\frac{\pi}{\pi}}$ & 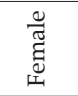 & a. & $\frac{\frac{0}{\pi}}{\sum^{\pi}}$ & $\sum^{\frac{\Perp}{\pi}}$ & a. & $\sum^{\frac{\pi}{\pi}}$ \\
\hline Life Stage & $\frac{\vec{z}}{\frac{\pi}{4}}$ & $\frac{\vec{z}}{\frac{7}{4}}$ & $\frac{⿱ ⺌}{\frac{7}{8}}$ & $\frac{\text { 吾 }}{\frac{0}{4}}$ & 蒙 & 訔 & 壱 & $\frac{\text { 壱 }}{\frac{0}{4}}$ & $\frac{\mathrm{H}}{\frac{\mathrm{z}}{4}}$ & 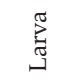 & 壱 \\
\hline $\begin{array}{l}\text { Synonim according to } \\
\text { Morse * }\end{array}$ & 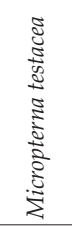 & 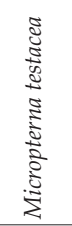 & 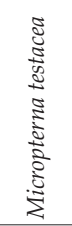 & 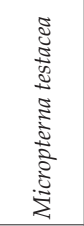 & 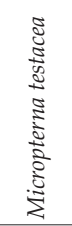 & & 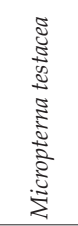 & 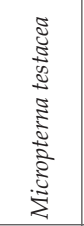 & & & 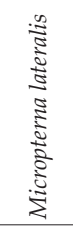 \\
\hline $\begin{array}{l}\text { Taxonomic designation } \\
\text { according to identifier }\end{array}$ & 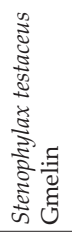 & 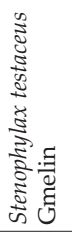 & 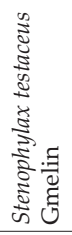 & 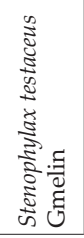 & 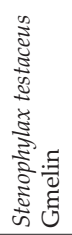 & 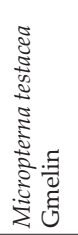 & 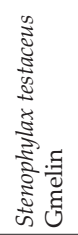 & 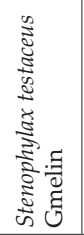 & 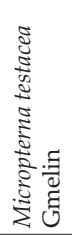 & 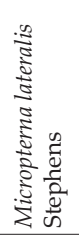 & 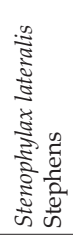 \\
\hline $\mathrm{mt} C O I$ haplotype & $\begin{array}{l}\text { I } \\
\hat{A}\end{array}$ & \multicolumn{4}{|c|}{ 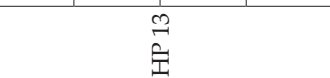 } & \multicolumn{3}{|c|}{$\begin{array}{l}\text { 亲 } \\
\dot{9}\end{array}$} & $\stackrel{20}{2}$ & 章 & 言 \\
\hline
\end{tabular}




\begin{tabular}{|c|c|c|c|c|c|c|c|c|c|c|c|c|c|c|}
\hline BOLD Sequence ID & 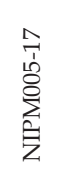 & 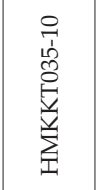 & 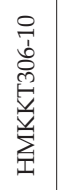 & $\begin{array}{l}\circ \\
\frac{1}{1} \\
\hat{0} \\
\stackrel{0}{\mid} \\
\sum_{1}^{\mid}\end{array}$ & 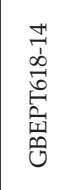 & 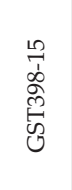 & 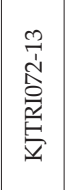 & 产 & 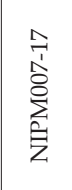 & 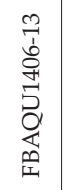 & 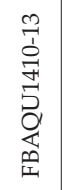 & 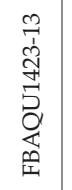 & 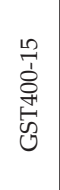 & $\begin{array}{l}\qquad 0 \\
\frac{1}{\alpha} \\
\alpha \\
6 \\
0 \\
0\end{array}$ \\
\hline Specimen ID & $\sum_{\exists}^{N_{1}}$ & 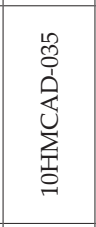 & 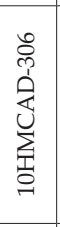 & 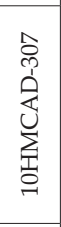 & $\begin{array}{l}\text { fि } \\
\text { Oे } \\
\hat{O} \\
0 \\
00 \\
0\end{array}$ & 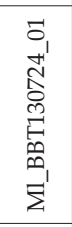 & 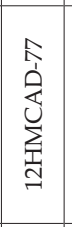 & $\bigcup_{\forall}^{N}$ & $\begin{array}{l}\vec{d} \\
\stackrel{d}{⿴ 囗 十} \\
\sum_{i}^{m}\end{array}$ & 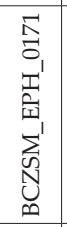 & 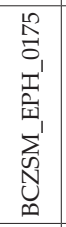 & 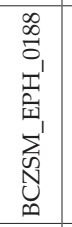 & 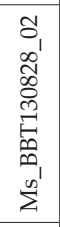 & 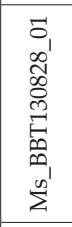 \\
\hline Location & 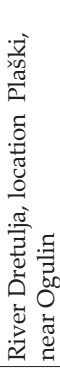 & 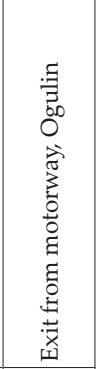 & 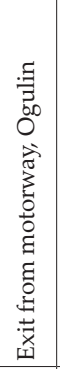 & 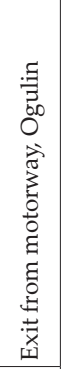 & 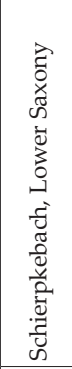 & 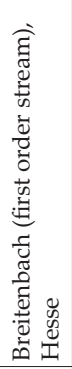 & 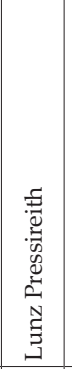 & 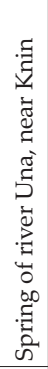 & 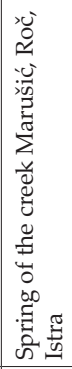 & 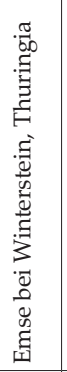 & 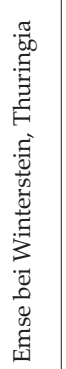 & 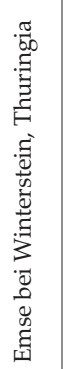 & 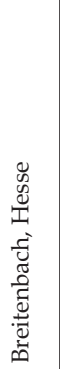 & 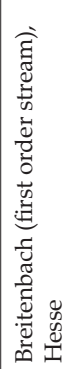 \\
\hline Country & $\begin{array}{l}\stackrel{\widetilde{\pi}}{\widetilde{J}} \\
\stackrel{U}{U}\end{array}$ & $\begin{array}{l}\stackrel{\widetilde{\pi}}{\widetilde{d}} \\
\stackrel{\mathscr{U}}{0}\end{array}$ & $\begin{array}{l}\widetilde{\widetilde{J}} \\
\stackrel{0}{0} \\
\tilde{U}\end{array}$ & 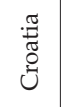 & 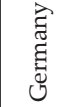 & 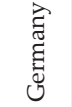 & $\begin{array}{l}\frac{\pi}{E} \\
\frac{5}{2} \\
\frac{2}{4}\end{array}$ & 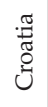 & 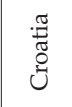 & 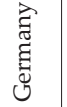 & 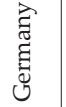 & 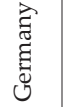 & $\begin{array}{l}\text { त्ञ } \\
\text { हूँ } \\
\text { Uु }\end{array}$ & 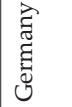 \\
\hline $\begin{array}{l}\text { Specimen morphologi- } \\
\text { cal identification by: }\end{array}$ & 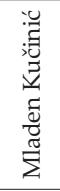 & 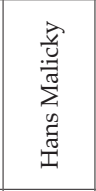 & 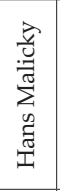 & 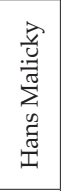 & 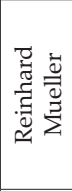 & 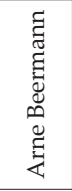 & 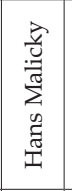 & 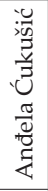 & 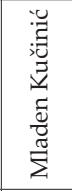 & 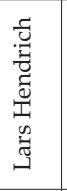 & 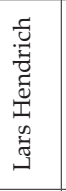 & 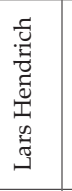 & 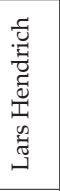 & 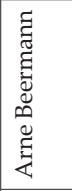 \\
\hline Sex & $\frac{0}{\pi^{\pi}}$ & a. & c. & a. & c. & c. & $\frac{0}{\pi}$ & 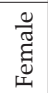 & $\sum_{\frac{\pi}{\pi}}^{\frac{0}{\Sigma}}$ & $\frac{0}{\pi}$ & $\sum^{\frac{\pi}{\pi}}$ & $\frac{0}{\pi^{\pi}}$ & $\sum_{\pi}^{\frac{0}{\pi}}$ & a. \\
\hline Life Stage & 蒙 & 蒙 & 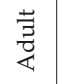 & $\frac{\vec{t}}{\vec{z}}$ & 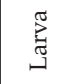 & 壱 & $\begin{array}{l}\frac{\mathrm{t}}{\mathrm{z}} \\
\frac{0}{4}\end{array}$ & $\frac{⿱ ⺌}{\frac{3}{8}}$ & $\frac{\vec{t}}{\frac{3}{3}}$ & $\begin{array}{l}\frac{ \pm}{\vec{z}} \\
\frac{0}{4}\end{array}$ & $\frac{\mathrm{t}}{\frac{3}{2}}$ & $\frac{\mathrm{t}}{\frac{\mathrm{J}}{4}}$ & $\frac{\overrightarrow{3}}{\vec{z}}$ & c. \\
\hline \multicolumn{15}{|l|}{$\begin{array}{l}\text { Synonim according to } \\
\text { Morse * }\end{array}$} \\
\hline $\begin{array}{l}\text { Taxonomic designation } \\
\text { according to identifier }\end{array}$ & 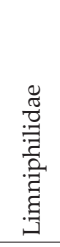 & 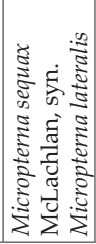 & 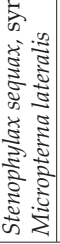 & 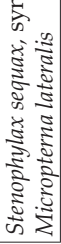 & 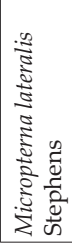 & 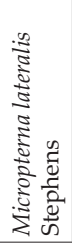 & 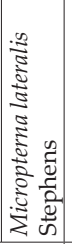 & 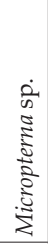 & 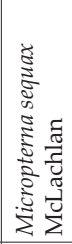 & 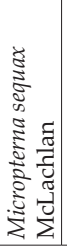 & 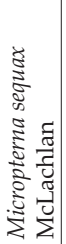 & 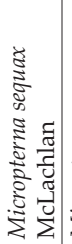 & 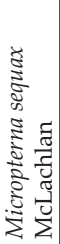 & 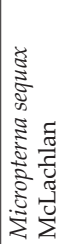 \\
\hline $\mathrm{mt} C O I$ haplotype & & & & $\stackrel{\infty}{\stackrel{1}{I}}$ & & & & 产 & $\begin{array}{l}\text { ลิ } \\
\hat{1}\end{array}$ & & & I & & \\
\hline
\end{tabular}




\begin{tabular}{|c|c|c|c|c|c|c|c|c|c|c|}
\hline BOLD Sequence ID & 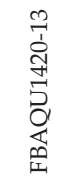 & 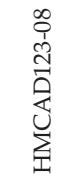 & 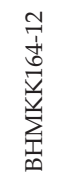 & 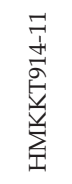 & 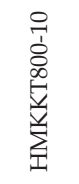 & 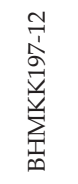 & 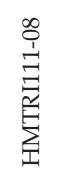 & 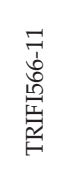 & 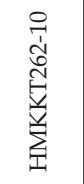 & 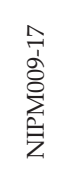 \\
\hline Specimen ID & 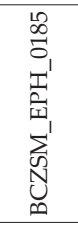 & 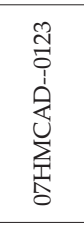 & 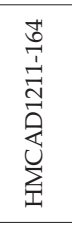 & 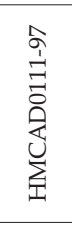 & 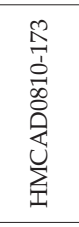 & 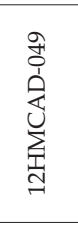 & 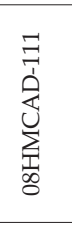 & 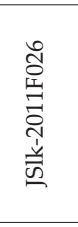 & 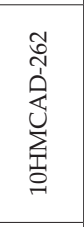 & 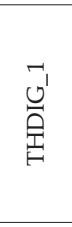 \\
\hline Location & 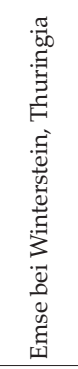 & 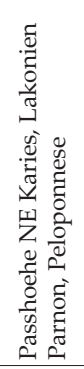 & 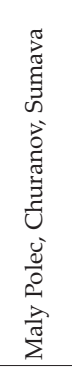 & 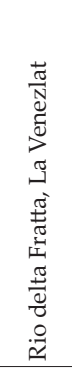 & 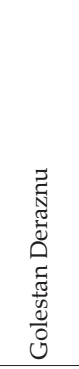 & 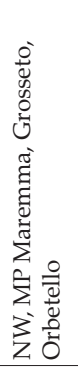 & 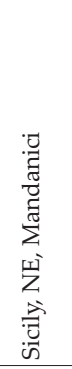 & 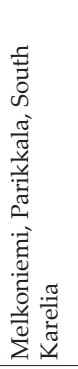 & 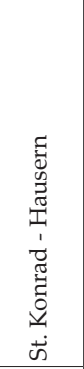 & 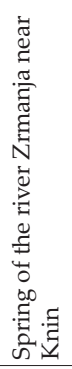 \\
\hline Country & 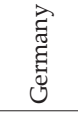 & 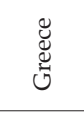 & 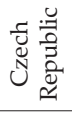 & ڤ & Ф્ञ్ & 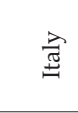 & 홒 & 胥 & 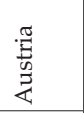 & 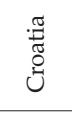 \\
\hline $\begin{array}{l}\text { Specimen morphologi- } \\
\text { cal identification by: }\end{array}$ & 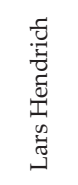 & 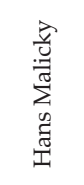 & 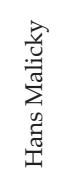 & 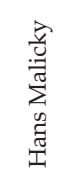 & 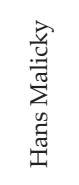 & 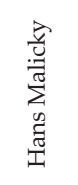 & 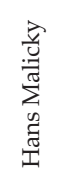 & 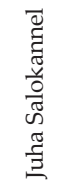 & 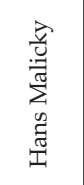 & 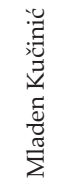 \\
\hline Sex & $\frac{\frac{\pi}{\pi}}{\frac{\pi}{4}}$ & 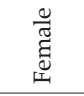 & c. & $\frac{\frac{\pi}{\pi}}{\frac{\pi}{\Sigma}}$ & $\frac{\frac{\Omega}{\pi}}{\sum^{\pi}}$ & c. & c. & 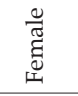 & r. & $\frac{\frac{\pi}{\pi}}{\sum}$ \\
\hline Life Stage & 莺 & 崇 & $\begin{array}{l}\frac{1}{\overrightarrow{5}} \\
\frac{0}{4}\end{array}$ & 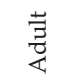 & 奇 & $\frac{n}{\frac{3}{5}}$ & 奇 & $\frac{\mathrm{U}}{\mathrm{z}}$ & $\frac{\overrightarrow{3}}{\frac{3}{2}}$ & $\frac{\overrightarrow{3}}{\frac{3}{4}}$ \\
\hline $\begin{array}{l}\text { Synonim according to } \\
\text { Morse * }\end{array}$ & & 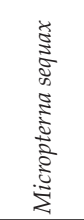 & 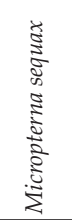 & 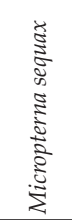 & & & & & & \\
\hline $\begin{array}{l}\text { Taxonomic designation } \\
\text { according to identifier }\end{array}$ & 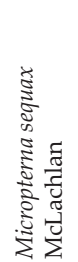 & 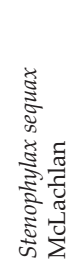 & 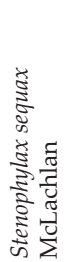 & 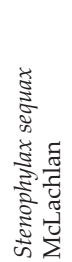 & 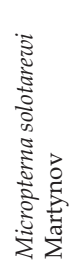 & 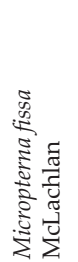 & 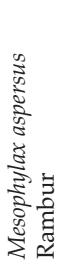 & 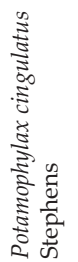 & 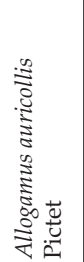 & 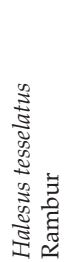 \\
\hline mt $C O I$ haplotype & $\begin{array}{l}\text { ה } \\
\hat{1}\end{array}$ & $\begin{array}{l}\stackrel{2}{2} \\
\text { I }\end{array}$ & $\begin{array}{l}\stackrel{ \pm}{N} \\
\vdots\end{array}$ & $\begin{array}{l}\stackrel{\text { ñ }}{1} \\
\text { 全 }\end{array}$ & $\begin{array}{l}\text { I } \\
\text { 主 }\end{array}$ & $\begin{array}{l}\hat{\Lambda} \\
\hat{I}\end{array}$ & & & & \\
\hline
\end{tabular}




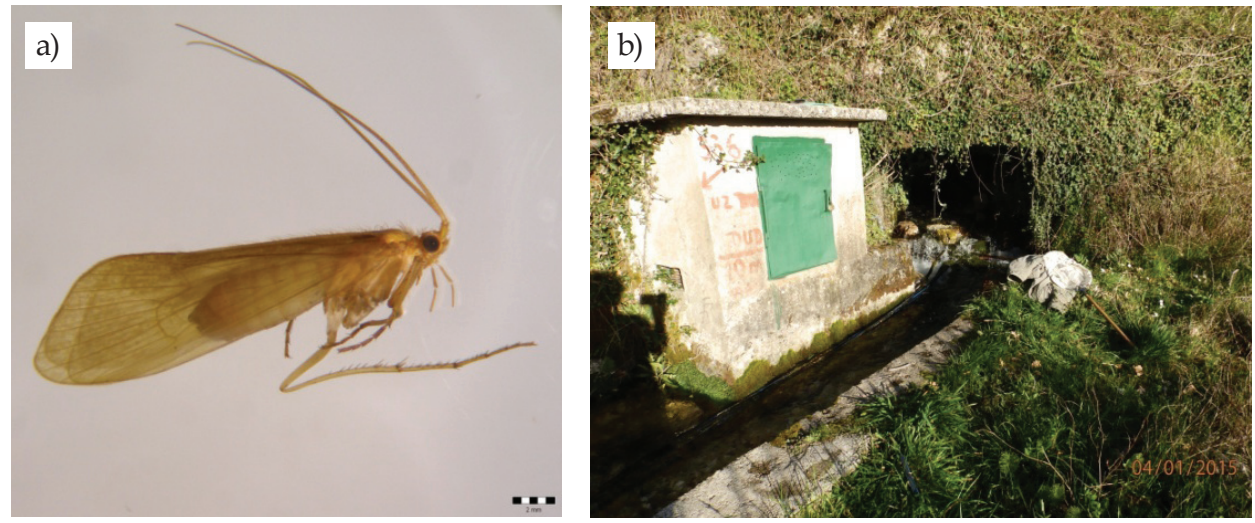

c)

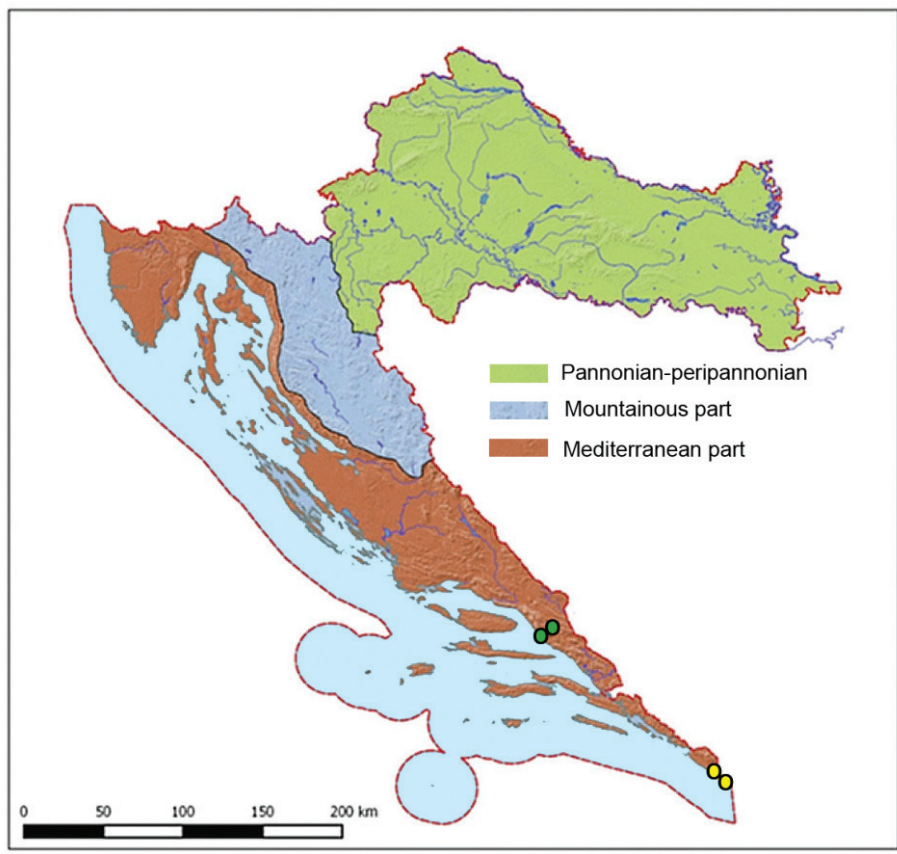

Fig. 1 A-C. Micropterna wageneri: A) adult (collected in Konavle region and deposited in the NIP-Trichoptera collection in the Croatian Natural History Museum in Zagreb); B) spring in the village Vodovađa (Konavle valley); C) geographical regions in Croatia according to BerTić et al. (2001), with locations where M. wageneri was found: Mt. Biokovo (green points) and Konavle region (yellow points).

\section{RESULTS AND DISCUSSION}

\section{Faunal data}

Micropterna wageneri (Fig. 1 A) was described by male adults (Fig. 2A) from Italy (Malicky, 1971). Female (Fig. 2B) was described by Moretti also from this region (MoreTTI, 1981). According to the literature data this species is distributed in southern Europe 
a)

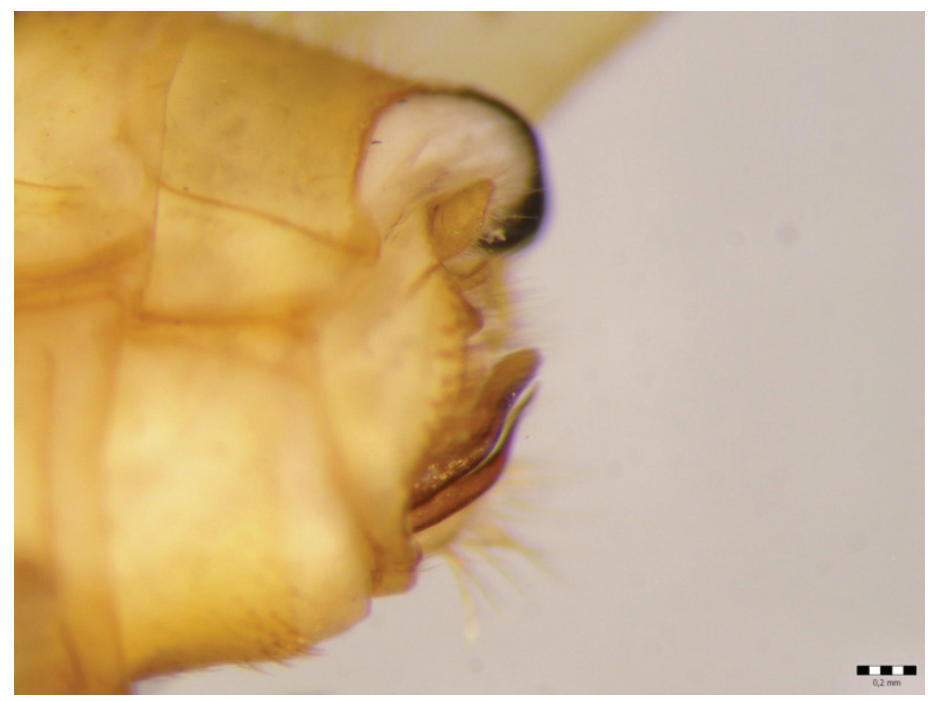

b)

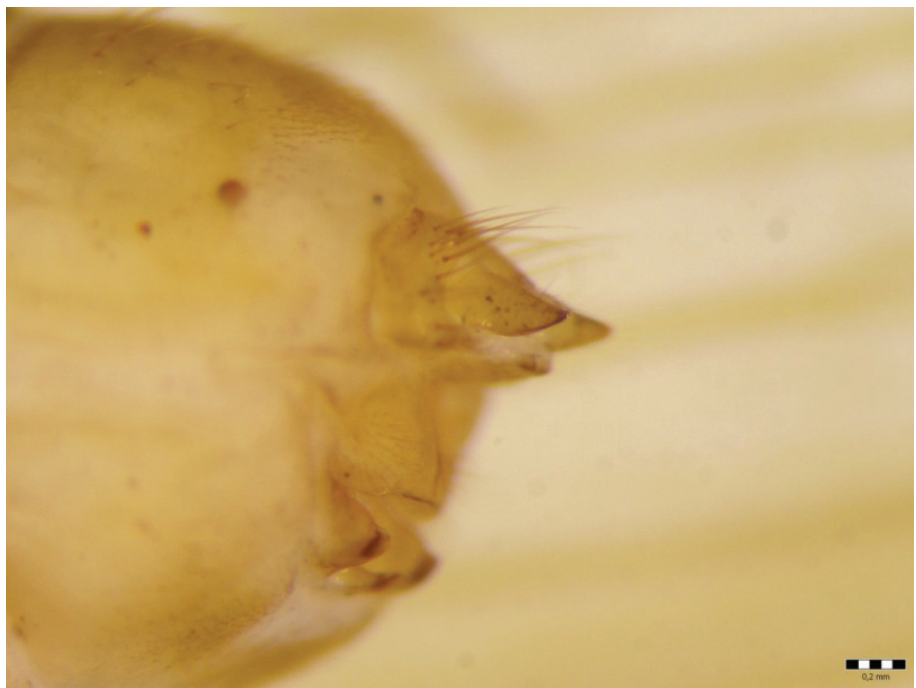

Fig. 2 A-B. Micropterna wageneri: A) male genitalia (left lateral view; B) female genitalia (left lateral view); specimens were collected in Konavle region and deposited in the NIP-Trichoptera collection in the Croatian Natural History Museum in Zagreb.

(Italy, Croatia, Albania...) (e.g. Malicky, 1971; Moretti, 1981, 1988; Cianficconi, 2002; OlÁH, 2010). Fauna Europaea reports this species from Serbia/Montenegro, however literature data for these two countries do not support these data. In the last 25 years we have visited Mt. Biokovo many times and collected a lot of species of butterflies and moths (e.g. Mladinov \& Kučinić, 1993; Kučinić et al., 1998; Minoci et al., 2011) and several species of caddisflies (M. Kučinić, unpublished data). Until our investigation in Konavle region in southern Croatia (region Dalmatia) $M$. wageneri was only known from two localities on Mt. Biokovo: first locality is at an altitude of $550 \mathrm{~m}$ (28 May 1995, 1 
male) and the second locality named Lađena at an altitude of $1270 \mathrm{~m}$ above sea level (28 May 1995, 2 males and 27 September 1995, 1 male). These specimens were collected 22 years ago (leg. M. Vajdić, F. Perović, M. Kučinić, det. H. Malicky) and are deposited in the Croatian Natural History Museum in Zagreb. During our investigation in Konavle region we collected $M$. wageneri at two locations, first one in a spring in the village Vodovađa ( $250 \mathrm{~m}$ a.s.l.) and the second one in the middle section of a stream near village Palje (150 m a.s.l.). Thus currently M. wageneri is known from four localities in Croatia, two on Biokovo Mt. and two in the Konavle region. Distance between these two areas is approximately 140 kilometres (Fig. 1 C).

\section{Molecular data}

The sequences obtained from $M$. wageneri samples submitted to the BOLD Identification Engine as well as to the GenBank did not find any match. Analysis of the genetic distance of DNA barcode region (mtCOI) between species of the genus Micropterna is shown in Tab. 2. Genetic difference among two specimens of M. wageneri (MW) is only $0.0019(0.2 \%)$. Intraspecific genetic difference within $M$. wageneri is identical to intraspecific difference $(0.2 \%)$ within $M$. testacea Gmelin. For comparison: ZHou (2009) reported the mean value of 0.48 for the intraspecific differences based on mtCOI barcode region (1481 sequence of 53 caddisfly taxa). This observed low value is in line with the observed variability of Drusus Stephens species (0.2\% in KučInIć et al., 2015).

The minimum interspecific difference between $M$. wageneri and other Micropterna species and outgroup species is 0.096 (9.6\%) (Tab. 2), which is above minimum difference between caddisfly species noted in literature (8.05\% in Smicridea species, Pauls et al., 2010; 8.2\% in Anisogamus species Graf et al., 2015). Most similar species to M. wageneri in this analysis are M. sequax McLachlan (TLIM_2) from Croatia and M. lateralis Stephens (GBEPT617-14) from Germany (0.096/9.6\%). Relatively same value of minimum interspecific difference for DNA barcode region is noted for Tinodes Curtis (10\% in KučInIć et al., 2016) and other Limnephilidae (8.2\% and 9.6\% in GrAF et al., 2015).

Micropterna wageneri specimens clustered within the well supported clade which is distinct from other Micropterna species (100\% BS support in both, ML and NJ analyses). The clustering of $M$. sequax sequences in three independent, well-supported clades in both NJ and ML analyses as well as in three distinct groups identified in ABGD analysis (group 2, group 3 and group 4 in Fig. 1) point to the existence of potentially new taxa within $M$. sequax. Often this kind of intraspecific divergence indicates the presence of cryptic species (Hebert et al., 2004). Clustering of M. lateralis with several M. sequax specimens as well as the sharing of the same COI haplotype between those two species (HP 18 haplotypes in Tab. 1) also prompts the need for further investigation of those taxa.

Thereby, population of these species from different parts of Europe should be analysed by means of morphological and more comprehensive phylogenetic analysis by employing additional molecular markers (mitochondrial as well as nuclear) to determine their exact taxonomic and phylogenetic status. In this case, DNA barcoding flagged possibly still unrecognised cryptic species complex. The use of morphological and molecular methods as a basis for integrative taxonomy (VIтEсек et al., 2017), as well as an additional analysis of ecological and biogeographic data, are very important aspect of modern taxonomic and phylogenetic research of such taxa whose status cannot be efficiently resolved without employing all mentioned approaches. 


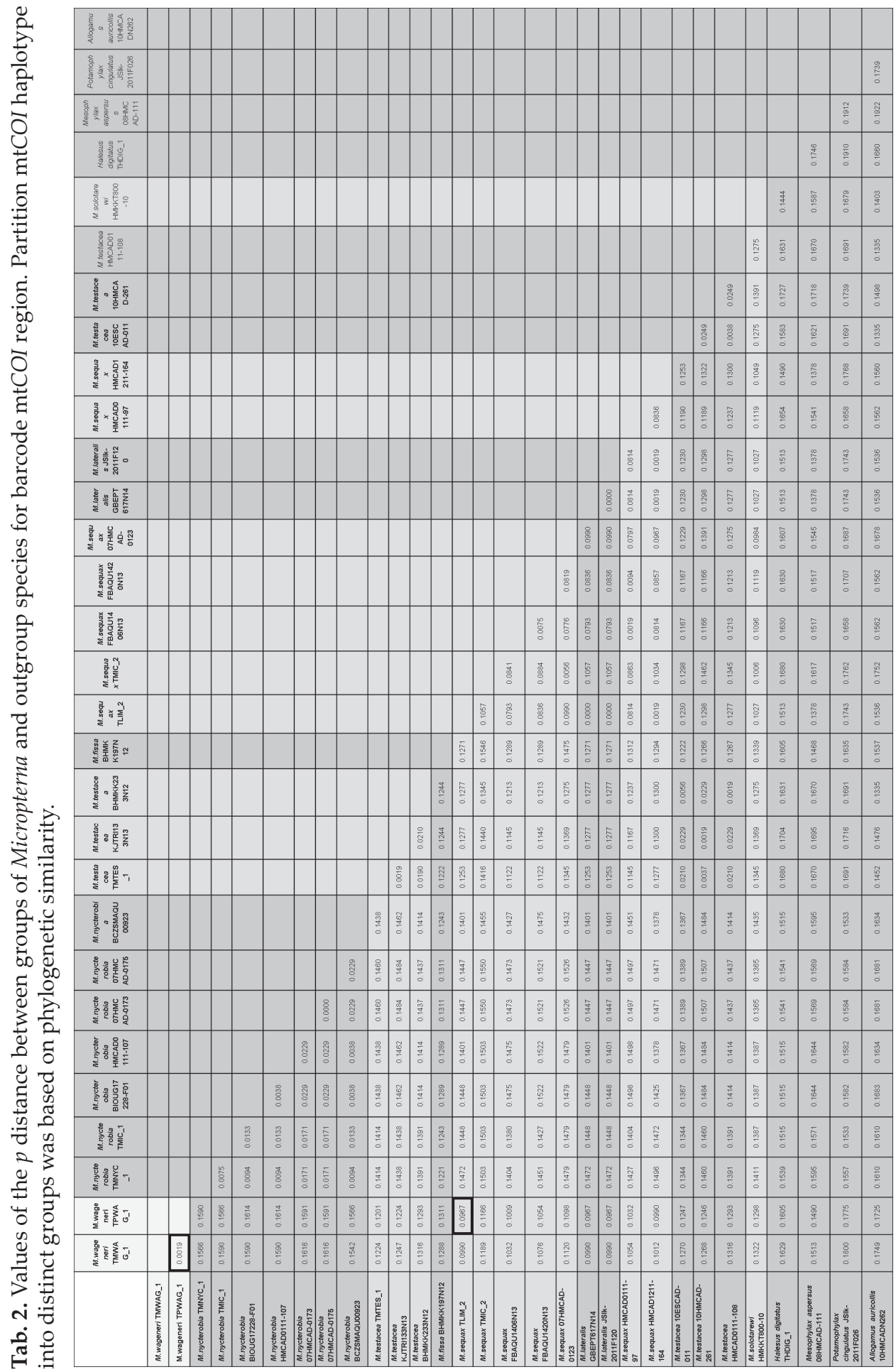




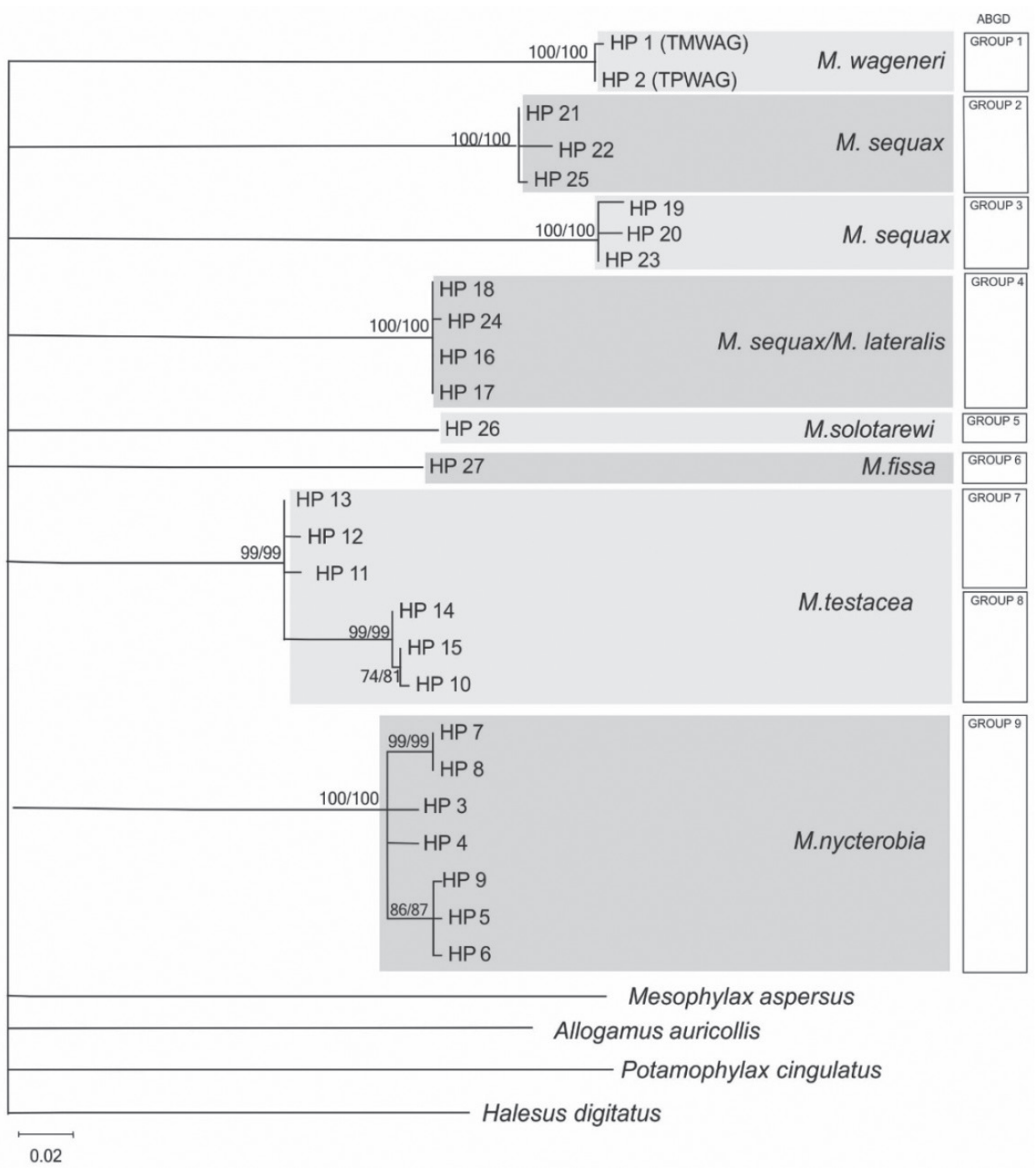

Fig. 3. Maximum likelihood (ML) phylogram based on $658 \mathrm{bp}$ long fragment of the DNA barcode region showing the relationships between species of the genus Micropterna. Numbers above the branches represent bootstrap support (BS) for Neighbor-Joining (NJ) and ML analysis (NJ/ML). BS values less than 70 are not shown. The groups delineated by Automatic Barcode Gap Discovery (ABGD) approach are shown on the right side of the tree.

The ABGD analysis revealed 9 genetic groups (Fig. 3). Interspecific distances of $M$. wageneri, among other specimen groups, did not overlap with intraspecific divergences as the ABGD analysis shows (Fig. 4). M. wageneri formed one group separated from other group of Micropterna and strongly indicates that $M$. wageneri is independent species. For 5 species from the genus Micropterna DNA barcoding analyses is done (Tab. $1,3)$. 


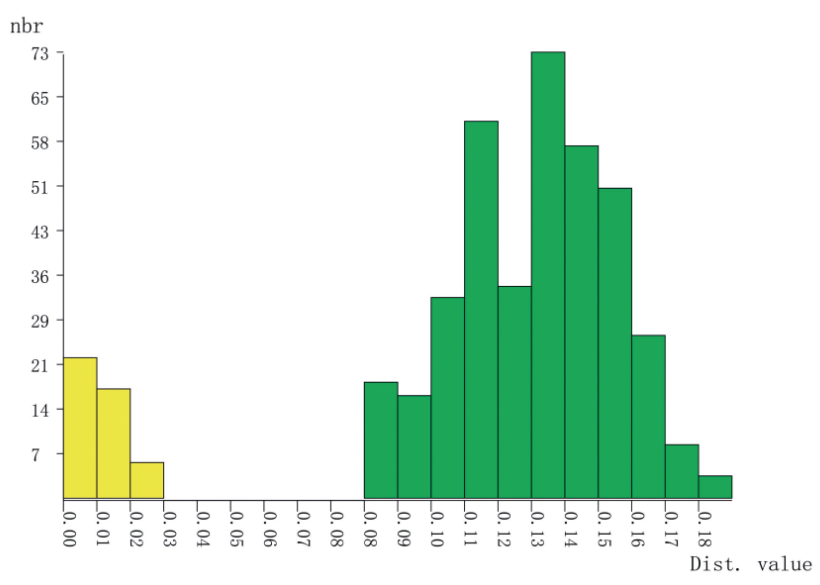

Fig. 4. Histogram depicting the frequency distribution of K2P distances for species of Liminiphilidae family used in this study, calculated by ABGD. The horizontal axis shows the pairwise K2P-distance, and the vertical axis shows the number of pairwise sequence comparisons. On the left side of histogram (yellow colour) is intraspecific and on right is interspecific (green colour) distance variation.

\section{Biodiversity of the genus Micropterna}

Thirty four species genus Micropterna are recorded in Europe, North Africa and West Asia (MALicky, 2004). In southeastern Europe (Balkan Peninsula) ten species from this genus are present: $M$. caesareica Schmid, M. coiffaiti Décamps, M. fissa McLachlan, $M$. lateralis Stephens, M. malaspina Schmid, M. nycterobia, M. sequax, M. testacea, M. taurica Martynov and M. wageneri (KumANSKI, 1988; Žrvić et al., 2002; MALICKY, 2004, 2005; IBRAhimi et al., 2013; OláH, 2010; Stanić-Koštroman et al. 2015;). In Croatian fauna 6 species have been found so far (e.g. LANGHOFFER, 1915; RADOvANOvić, 1935; MARINKOvićGospodnetić, 1979; Kučinić \& Ilić, 1993; Kučinić, 2002; Cerjanec, 2012) (Tab. 3). The

Tab. 3. Biodiversity and distribution of the genus Micropterna in three geographical regions in Croatia (*species which have DNA barcoding analysis from specimens collected in Croatia), some DNA barcoding data are unpublished.

\begin{tabular}{|l|c|c|c|}
\hline Species of genus Micropterna & $\begin{array}{c}\text { Pannonian- } \\
\text { peripannonian }\end{array}$ & $\begin{array}{c}\text { Mountainous part } \\
\text { part of Croatia }\end{array}$ & $\begin{array}{c}\text { Mediterranean part } \\
\text { of Croatia }\end{array}$ \\
\hline M. fissa McLachlan & - & - & $\bullet$ \\
\hline M. lateralis Stephens* & $\bullet$ & $\bullet$ & - \\
\hline M. nycterobia McLachlan* & - & $\bullet$ & $\bullet$ \\
\hline M. sequax McLachlan* & $\bullet$ & $\bullet$ & $\bullet$ \\
\hline M. testacea Gmelin* & - & $\bullet$ & $\bullet$ \\
\hline M. wageneri Malicky* & - & - & - \\
\hline TOTAL & 2 & 4 & 5 \\
\hline
\end{tabular}


highest number represented by 5 species was recorded in the Mediterranean part of Croatia, while in the mountain area there are 4 recorded species and in the Pannonianperipannonian part of Croatia only two species (Tab. 3). The species that appears in all three biogeographic regions of Croatia is the species M. sequax (Tab. 3). Two species recorded are distributed only in the Mediterranean area: $M$. fissa and M. wageneri (Tab. 3). Three species were recorded in two regions of Croatia: M. lateralis (Pannonian-peripanonian and mountainous part), M. nycterobia and M. testacea (mountainous and Mediterranean part) (Tab. 3).

\section{Ecological data}

According to Graf et al (2008) Micropterna wageneri belongs to submountain and mountain species. During our investigations of Mt. Biokovo, we found M. wageneri in middle and high altitudes (550 $\mathrm{m}$ and $1270 \mathrm{~m}$ above sea level) but in Konavle region we found this species at significantly lower altitudes $(220 \mathrm{~m}$ and $250 \mathrm{~m}$ above sea level). These results were expected because this Mediterranean species probably has the ability to migrate into higher mountain regions, like some other limnephilid species (e.g. NovÁк \& SEHNAL, 1963). There are no natural aquatic habitats, such as springs or small streams, in higher mountainous areas of Mt. Biokovo. These types of aquatic habitats were established only at lower altitudes on Biokovo, where M. wageneri has not been established yet. In Konavle region M. wageneri probably inhabits springs and small streams, habitats in which we have collected adults. In small manmade canals in the Konavle valley we found interesting aquatic insect fauna (M. Kučinić unpublished data).

The larva of M. wageneri is not described yet (WARINGER \& GRAF, 1997, 2011; WARINGER \& MALICKY, 2016). Future investigations of caddisflies in the Konavle region will be focused on finding and possibly describing morphological features of larvae of this species. Very interesting ethological and ecological feature of the genus Micropterna is the ability of adults to live in pits and caves like troglophilic species (if such a habitat type exists in that area). The first finding of the genus Micropterna in Croatian subbteranean habitats is from the beginning of the XX. century, with the species: M. lateralis, M. nycterobia and M. sequax (e.g. Radovanović, 1935; LANGHoffer, 1915; GotTstein et al., 2002; Kučinić et al., 2012). The fourth species from the genus Micropterna in subterranean habitats in Croatia was a species M. testacea (Kučinić \& ILIĆ, 1993). This species was found on Mt. Biokovo for the first time for Croatian fauna (Kučınić \& ILIĆ, 1993). Caddisflies are frequent faunistic element in caves and pits of the Dinaric karst, although in a relatively small number of species (GotTstein-MAтоčEC, 2002; Kučinić et al., 2012, M. KučInIĆ unpublished data). So far 12 species have been found in the fauna of Croatia (GotTstein-MAtočEc, 2002; Kučinić et al., 2012). They belong to the group of troglophilic organisms that spend only one period of their life cycle in subterranean habitats. The most common species of caddisflies in speleological objects in Croatia are M. sequax, $M$. nycetrobia and Stenophylax permistus McLachlan (GotTstein et al., 2002; Kučinić et al., 2012). Micropterna wageneri was not found in these types of habitats in Croatia, but Moretti recorded this species as element of subterranean fauna in Italy (MoreTTI, 1988). The reason why caddisflies are entering speleological objects are not entirely clear. The occurrence of this two genera in caves (Micropterna and Stenophylax Kolenati) is not accidental and that has been proved by overwhelming number of the finds so far (e.g. Gottstein-Matočec et al., 2002; Kučinić et al., 2012). We can assume that they found shelter in subterranean habitats. The biological reasons behind the migration of species of the genera Micropterna and Stenophylax to speleological objects remain to be found. 


\section{ACKNOWLEDGMENTS}

This work has been supported in part by the Croatian Science Foundation under the project (IP-2016-06-9988, DNA Barcoding of Diversity of Croatian Fauna, by M. Kučinić) and by the "EU Natura 2000 Integration Project (NIP)" funded by the Croatian Ministry of Environmental and Nature Protection and University of Zagreb. For tehnical help authors thank Prof. Petar Kružić from the Faculty of Science, University of Zagreb, and also two anonymous reviewers.

Received June 5, 2017

\section{REFERENCES}

Bertić I., Lampek Pavčnik, I. \& Radovinović, R., 2001. Satelitski atlas Hrvatske. Naklada Ljevak i Gisdata, Zagreb, 360 pp.

Bickford, D., Lohman, D.J., Sodhi, N.S., NG, P.K.L., Meier, R., Winker, K., Ingram , K.K. \& Das, I., 2007 : Cryptic species as a window on diversity and conservation. Trends in Ecology and Evolution 22, 148-155.

Casale, A. \& Jalžıć, B., 1988: Radziella (new genus) styx n. sp., a new exceptional troglobitic Bathysciinae (Coleoptera, Catopidae) from the Dinaric Region, Jugoslavia. Boll. Mus. Reg. Sci. Nat. 6 (2), 349-358.

CERJANEC, D., 2012: Ekološke i biogeografske značajke faune tulara (Insecta: Trichoptera) u različitim tipovima staništa sliva rijeke Dobre. Doktorska disertacija (PhD Thesis), Faculty of Science, University of Zagreb, 172 pp. (in Croatian)

Cianficconi, F., 2002: The third list of Italian Trichoptera (1990-2000). Proceedings of the $10^{\text {th }}$ International Symposium on Trichoptera (Ed. Mey, W.) Nova Supplementa Entomologica Keltern 15, 349-358.

Dincă, V., Wiklund, C., Lukhtanov, V.A., Kodandaramaiah, U., Norén, K., Dapporto, L., Wahlberg, N., Vila, R. \& Friberg, M., 2013: Reproductive isolation and patternsof genetic differentiation in a cryptic butterfly species complex. Journal of Evoutionary Biology 26, 2095-2106.

Folmer, O., Black, M., Hoen, W., Lutz, R. \& Vrijenhoek, R., 1994: DNA primers for amplification of mitochondrial cytochrome c oxidase subunit I from diverse metazoan invertebrates. Molecular marine biology and biotechnology 3, 294-299.

Gottstein-Matočec, S., Bakran-Petricoli, T., Bedek, J., Bukovec, D., Buzjak, S., Franičević, M., Jalžić, B., Kerovec, M, KletečKi, E., Kovačić, M., Kralj, J., Kružić, P., Kučinić, M., Kuhta, M., Matočec, N., Ozimec, R., RaĐa, T., Štamol, V., Ternjej, I. \& Trvtković, N., 2002: An overview of the cave and interstitial biota of Croatia. Natura Croatica 11 (Suppl. 1), 1-112.

Graf, W., Murphy, J., Dahl, J., Zamora-Muñoz, C. \& López-Rodríguez, M.J., 2008: Distribution and ecological preferences of European freshwater organisms. Volume 1. Trichoptera. Pensoft, Sofia-Moscow, 388 pp.

Graf, W., Vitecek, S., Previšić, A. \& Malicky, H., 2015: New species of Limnephilidae (Insecta: Trichoptera) from Europe: Alps and Pyrenees as harbours of unknown biodiversity. Zootaxa 3911 (3), 381-395. http://dx.doi.org/10.11646/zootaxa.3911.3.5

HabdijA, I., 1989: Trophic importance of trichopterous larvae in benthos oft he lakes Plitvice. Periodicum Biologorum 90 (3), 355-361.

Habdija, I., Primc-Habdija, B., Matoničkin, R., Kučinić M., Radanović, I., Miliša, M. \& Mihaljević, Z., 2004: Current velocity and food supply as factors affecting the composition of macroinvertebrates in bryophyte habitats in karst running water. Biologia 59 (5), 577-593.

Hall, T.A., 1999: BioEdit. A user-friendly biological sequence alignment editor and analysis program for Windows 95/98/NT. Nucl. Acids Symp. Ser. 41, 95-98.

Hebert, P.D.N., Cywinska, A., Ball, S.L. \& DeWAard, J.R., 2003a: Biological identifications through DNA barcodes. Proc. R. Soc. Lond. B. Biol. Sci. 270, 313-322.

Hebert, P.D.N., Ratnasingham, S. \& DeWaArd, J.R., 2003b: Barcoding animal life: Cytochrome c oxidase subunit 1 divergences among closely related species. Proc R Soc Lond B Biol Sci 270, S596-S599.

Hebert, P. D., Penton, E. H., Burns, J. M., Janzen, D. H., \& Hallwachs, W., 2004: Ten species in one: DNA barcoding reveals cryptic species in the neotropical skipper butterfly Astraptes fulgerator. Proc. Natl. Acad. Sci. U.S.A. 101 (41), 14812-14817. 
Ibrahimi, H., Gashi, A., Grapci-Kotori, L. \& Kučinić, M., 2013: First records of the genus Micropterna Stein, 1873 (Insecta: Trichoptera) in Kosovo with distributional and ecological notes. Natura Croatica 22 (1), 147-155.

KRULIK, I., 1979: Biomasa zoonentosa Krških Rijeka. Drugi kongres ekologa Jugoslavije. II (knjiga radova). Savez Društava Ekologa Jugosla. (eds. ČanaK, M., Ilijanić, Lj., Gradojević, Z., Pejčinović, D., PopovsKa, O., Rauš, Đ., Stefanović, V., Žušančić, M., ŽunjIĆ, K.), 1813-1827.

KRUŠNiK, C. \& URbanič, G., 2002: Preliminary List of Slovenian Trichoptera. Proceedings of the $10^{\text {th }}$ International Symposium on Trichoptera (Ed. Mey W.). Nova Supplementa Entomologica 15, 359-364.

KučinIć, M., 2002: Raznolikost i rasprostranjenost tulara (Trichoptera, Insecta) u staništima Plitvičkih jezera (Biodiversity and distribution of caddisflies (Trichoptera, Insecta) of Plitvice Lakes. Doktorska disertacija (PhD Thesis) (in Croatian), University of Zagreb, Faculty of Science, pp. 138.

Kučinić, M. \& ILIć, D., 1993: Micropterna testacea Gmelin 1789 (Insecta, Trichoptera) nova vrsta u fauni tulara Republike Hrvatske. Rad HAZU 26, 125-131.

KučinIĆ, M., JALžIĆ, B. \& Pelić, D., 1998: Xylocampa areola (Esper, 1789), Eurois occulta (Linnaeus, 1758) and Euxoa decora (Denis \& Schiffermüller, 1775), new elements in the noctuid fauna (Insecta: lepidoptera: Noctuidae) of Croatia. Natura Croatica 7 (3), 213-226

Kučinić, M. \& MaLicky, H., 2002: Rhyacophila dorsalis plitvicensis new subspecies from Croatia. Proceedings of the $10^{\text {th }}$ International Symposium on Trichoptera (Ed. Mey W.). Nova Supplementa Entomologica 15, 145-147.

Kučinić, M., Previšić, A., Gottstein, A., Hrašovec, B., Stanić-Koštroman S., Pernek, M. \& Delić, A., 2008: Description of the larvae of Drusus radovanovici septentrionis Marinković-Gospodnetić, 1976 and Drusus croaticus Marinković-Gospodnetić, 1971 (Trichoptera: Limnephilidae) from Bosnia and Herzegovina and Croatia. Zootaxa 1783, 1-17.

Kučinić, M., VučKović, I., KutnjaK, H., Šerić-Jelaska, L. \& Marguš, D., 2011: Diversity, distribution, ecology and biogeography of caddisflies (Insecta: Trichoptera) in the Krka River (National Park "Krka", Croatia). Zoosymposia 5, 255-268.

KuČINIĆ, M., OzımEc, R. \& JaLžıć, B., 2012: Diversity and distribution of troglophilic fauna of caddisflies and moths (Insecta: Trichoptera, Lepidoptera) of Croatia. In eds. Kováč, L., Uhrin, M., Mock, A. \& LuptÁčıK, P.: 21 st International Conference of Subterranean Bilogy (Košice 2-7 September 2012). Abstract book, 61-62.

Kučinić, M., Szivák, I., PAuls, S.U., BÁLInt, M., Delić, A. \& VučKović I., 2013: Chaetopteryx bucari sp. n., a new species from the Chaetopteryx rugulosa group from Croatia (Insecta, Trichoptera) with some molecular, taxonomical and ecological notes on the group. ZooKeys 320, 1-28. doi: 10.3897/zookeys.320.4565

Kučinić, M., Previšić, A., Graf, W., Mihoci, I., Šoufek, M., Stanić-Koštroman, S., Lelo, S., Vitecek, S. \& WARINGER, J., 2015: Larval description of Drusus bosnicus Klapálek 1899 (Trichoptera: Limnephilidae), with distributional, molecular and ecological features. Zootaxa 3957(1), 85-97.

Kučinić, M., Ćukušić, A., Podnar, M., Landeka, M., Plavec, H., Plantak, M., Akimbekova, N. \& Žalac, S., 2016: The first record of Tinodes antonioi Botosaneanu \& Taticchi-Viganò, 1974 (Insecta, Trichoptera) in Croatia with DNA barcoding and ecological data and notice of biodiversity and distribution of the genus Tinodes in Croatia. Natura Croatica 25 (1), 131-149.

Kumanski, K. P., 1988: Trichoptera, Integripalpia. Fauna Bulgarica 19. Bulgarska Akademi na Naukite, Sofia, $353 \mathrm{pp}$.

Kumar, S., Stecher, G. \& Tamura, K., 2016: MEGA7: Molecular Evolutionary Genetics Analysis version 7.0 for bigger datasets. Mol. Biol. Evol. 33 (7), 1870-1874.

LANGHoffer, A., 1915: Fauna hrvatskih pećina (spila). II. (Fauna cavernarum Croatiae, II). Prirodoslovna istraživanja Hrvatske i Slavonije 7, 3-22.

LinNAeus, C., 1758: Systema naturae per regna tria naturae: secundum classes, ordines, genera, species, cum characteribus, differentiis, synonymis, locis (in Latin) $\left(10^{\text {th }}\right.$ ed.). Stockholm: Laurentius Salvius.

Malicky, H., 1971: Eine neue Micropterna (Trichoptera, Limnephilidae) aus Italien - mit einem überblick über die Gattungen Stenophylax, Micropterna und Mesophylax? Die Höhle (Wien) 22 (1), 15-19.

Malicky, H., 2004: Atlas of European Trichoptera. Springer, Dordrecht, 359 pp.

Malicky, H., 2005: Die Köcherfliegen Griechenlands. Denisia 17, 1-240.

Marinković-Gospodnetić, M., 1979: Trichoptera (Insecta) velikih karstnih izvora u Dinaridima. Savez Društava Ekologa Jugoslavije. (eds. ČanaK, M., Ilijanić, LJ., Gradojević, Z., Pejčınović, D., PopovsKa, O., Rauš, Đ., Stefanović, V., Žušančić, M., ŽunjIĆ, K.), 1837-1849. 
Matić, N., Maldini, K., Tomas, D., Ćuk, R., Milović, S., Miklavčić, I. \& ŠIrac, S., 2016: Geochemical characteristics of the Gacka river karstic springs (Dinaric karst, Croatia) with macroinvertebrate assemblages overview. Environmental Earth Science 75, 1308.

MatoničKin, I., 1987: Građa za limnofaunu krških voda tekućica Hrvatske. Biosistematika 13 (1), 25-35.

MatoničKin, I. \& PAvletić, Z., 1967: Hidrologija potočnog sistema Plitvičkih jezera i njegove biocenološke značajke. Krš Jugoslavije 5, 83-126.

MatoničKin, I., Pavletić, Z., TAvčAR, V. \& KrKač, N., 1971: Limnološka istraživanja reikotioopa i fenomena protočne travertinizacije u Plitvičkim jezerima. Prirodoslovna istraživanja JAZU 40, Acta Biologica 7(1), 1-88.

Minoci, I., Hršak, V., Kučinić, M., Mičetić Stanković, V., Delić, A. \& Tvrtković, N., 2011: Butterfly diversity and biogeography on the Croatian karst mountain Biokovo: Vertical distribution and preference for altitude and aspect? European Journal of Entomology 108 (4), 623-633.

Mladinov, L. \& Kučinić, M., 1993: Preliminarni podaci za faunu sovica (Lepidoptera, Noctuidae) planine Biokovo (Hrvatska). Acta Biokovica 6, 33-45.

Moretri, G., 1981: New Trichoptera Species and Subspecies Found in Italy. Proceedings of the Third International Symposium on Trichoptera (ed. Moretri, G.), Seria Entomologica 20, 1-14.

Moretri, G., 1988: Tricotteri del Museo Civico di Scienze Naturali „Enrico Caffi“ di Bergamo. Riv. del Museo Civico di Scienze Naturali „Enrico Caffi“ di Bergamo 13, 1-19.

Morse, J.C., 2017: Trichoptera World Checklist (Ed. Morse, J.C.). http://entweb.clemson.edu/database/ trichopt/index.htm (accessed 24 May 2017)

Novák, K. \& Sennal, F., 1963: The development cycle of some species of the genus Limnephilus (Trichoptera). Časopis Československe Společnosti Entomologické 60 (1-2), 68-80.

OLÁH, J., 2010: New species and new records of Palearctic Trichoptera in the material of the Hungarian Natural History Museum. Annales Historico-Naturales Musei Nationalis Hungarici 102, 65-117.

Pauls, S.U., Blahnik, R.J., Zhou, X., Wardwell, C.T. \& Holzenthal, R.W., 2010: DNA barcode data confirm new species and reveal cryptic diversity in Chilean Smicridea (Smicridea) (Trichoptera: Hydropsychidae). J. N. Am. Benthol. Soc. 29 (3), 1058-1074. doi: 10.1899/09-108.1

Previšić, A., Graf, W., Vitecek, S., Kučinić, M., Bálint, M., Keresztes, L., Pauls, S.U. \& Waringer, J., 2014: Cryptic diversity of caddisflies in the Balkans: the curious case of Ecclisopteryx species (Trichoptera: Limnephilidae). Arthropod Syst. Phylogeny 72 (3), 309-329.

Previšić, A., Kerovec , M. \& Kučinić, M., 2007: Emergence and Composition of Trichoptera from Karst Habitans, Plitvice lakes Region, Croatia. International Review of Hydrobiology 92 (1), 61-83.

Previšić, A., Walton, C., Kučinić, M., Mitrikeski, P.T. \& Kerovec, M., 2009: Pleistocene divergence of Dinaric Drusus endemics (Trichoptera, Limnephilidae) in multiple microrefugia within the Balkan Peninsula. Mol. Ecol. 18 (4), 634-647. doi: 10.1111/j.1365-294X.2008.04046.x

Puillandre, N., Lambert, A., Brouillet, S. \& Achaz, G., 2012: ABGD, Automatic Barcode Gap Discovery for primary species delimitation. Molecular Ecology 21 (8), 1864-1877.

Radovanović, M., 1935: Trihoptere Jugoslavije. Glasnik Zemaljskog Muzeja u Bosni i Hercegovini 47, 73-84.

Ratnasingham, S. \& Hebert, P.D.N., 2007: BOLD - The Barcode of Life Data System (www.barcodinglife. org). Molecular Ecology Notes 7, 355-364. doi:10.1111/j.1471-198286.2006.01678.x

Ross, H.H., 1974: Biological Systematics. Addison-Wesley Publishing Company Inc., Reading, Massachusetts, pp. 345.

Shtinkov, N., Kolev, Z., Vila R. \& Dincă, V., 2016: The sibling species Leptidea juvernica and L. sinapis (Lepidoptera, Pieridae) in the Balkan Peninsula: ecology, genetic structure, and morphological variation. Zoology 119, 11-20. http://dx.doi.org/10.1016/j.zool.2015.12.003

Stanić-Koštroman, S., Previšić, A., Planinić, A., Kučinić, M., ŠKobić, D., Dedić, A. \& Durbešić, P., 2015 : Environmental determinants of contrasting caddisfly (Insecta, Trichoptera) biodiversity in the Neretva and Bosna River basins (Bosnia and Herzegovina) under temperate and Mediterranean climates. International Review of Hydrobiology 100 (2), 79-95.

Szivák, I., Mikes, T., Szalontai, B., Kučinić, M., Vučković, M., Vadkerti, E., Kisfali, P., Pauls, U.S. \& BÁLINT, M., 2013: Ecological divergence of Chaetopteryx rugulosa species complex (Insecta, Trichoptera) linked to climatic niche diversification: Hydrobiologia 794 (1), 31-47. doi:10.1007/s10750-016-3068-0

Villesen, P., 2007: FaBox: an online toolbox for fasta sequences, Molecular Ecology Notes 7 (6), $965-968$. doi:10.1111/j.1471-8286.2007.01821.x 
Vitecek, S., Kučinić, M., Previšić, A., Žıvić, I., Stojanović, K., Kereszztes, L., BÁlint, M., Hoppeler, F., Waringer, J., Graf, W., Steffen U. \& Pauls, S.U., 2017: Integrative taxonomy by molecular species delimitation: multi-locus data corroborate a new species of Balkan Drusinae micro-endemics. BMC Evolutionary Biology 17 (129), 1-18. DOI 10.1186/s12862-017-0972-5

VučKović, I., Previšić, A. Graf, W. \& Kučinić, M., 2011: Description of the female anddistribution of Annitella apfelbecki Klapálek, 1899 (Insecta: Trichoptera). Aquatic Insects 33 (4), 381-389.

WARINGER, J. \& GRAF, W., 1997: Atlas der Österreichischen Köcherfliegenlarven. Facultus Universitätsverlag, Wien, $286 \mathrm{pp}$.

WARINGER, J. \& Graf, W., 2011: Atlas der mitteleuropäischen Köcherfliegenlarven - Atlas of Central European Trichoptera Larvae. Erik Mauch Verlag, Dinkelscherben, 468 pp.

Waringer, J. \& Malicky, H., 2016: The larvae of Micropterna coiffaiti Décamps 1963, Micropterna taurica Martynov 1917, and Potamophylax goulandriorum Malicky 1974 (Trichoptera: Limnephilidae), including a key to the hitherto known Stenophylacini larvae of the Hellenic western Balkan region. Zootaxa 4175 (1), 43-56.

Zноu, X., 2009: The larvae of Chinese Hydropsychidae (Insecta: Trichoptera), Part I: Arctopsyche shimianensis, Parapsyche sp. A, and Diplectrona obscura. Zootaxa 2174, 1-17.

Žıvić, I., Marković, Z. \& Brajković, M., 2002: First check list of Serbian Trichoptera. Folia HistoricoNaturalia Musei Matraensis 26, 269-277. 